\title{
Relación de los niveles de fuerza del tren superior con la calidad de vida en población adulta mayor europea
}

\author{
$\mathrm{M}^{\mathrm{a}}$ Helena Vila ${ }^{1}$, Irimia Mollinedo ${ }^{1}$, y José Cancela ${ }^{2}$ \\ ${ }^{1}$ Universidad de Vigo (España); ${ }^{2}$ Instituto de Investigación Sanitaria \\ Galicia Sur, Sergas (España)
}

El papel de la fuerza muscular en el desempeño de las actividades de la vida diaria y el ejercicio, así como en la prevención de enfermedades crónicas, se reconoce cada vez más. El objetivo de este estudio fue analizar los niveles de fuerza del tren superior con la calidad de vida de la población europea mayor de 65 años. Fueron reclutados 419 personas mayores (69.12 \pm 9.19 edad) de diferentes países Europeos (Bulgaria, España, Hungría, Italia y Portugal), pertenecientes al proyecto Europeo IN COMMON SPORTS Erasmus Plus. Se valoró la fuerza de miembros superiores por medio del Handgrip de ambos brazos y la calidad de vida a través del test EuroQol-5D (EQ-5D). Se realizó un análisis descriptivo y una correlación de Pearson. Los resultados constatan una relación inversa entre calidad de vida y fuerza de tren superior, es decir, mayores valores de fuerza de tren superior se relacionan con mejor calidad de vida para la población europea mayor de 65 años.

Palabras clave: Fuerza, autonomía, actividad física, dinamómetro, envejecimiento activo.

Relationship between upper limbs strength levels and the quality of life in the European elderly population. The role of muscle strength in the performance of activities of daily living and exercise, as well as in the prevention of chronic diseases, is increasingly recognized. The objective of this study was to analyze the strength levels of the upper limbs with the quality of life in the European population over 65 years. 419 elderly people $(69.12 \pm 9.19$ years $)$ were recruited from different European countries (Bulgaria, Spain, Hungary, Italy and Portugal), belonging to the European project IN COMMON SPORTS Erasmus Plus. The strength of the upper limbs was assessed by means of the Handgrip of both arms and the quality of life through the EuroQol-5D test (EQ-5D). A descriptive analysis and a Pearson correlation was performed. The results show an inverse relationship between quality of life and higher train strength, that is, higher values of higher train force are related to better quality of life for the European population over 65 years.

Keywords: Strength, funcionality, physical activity, handgrip, active aging.

Correspondencia: $\mathrm{M}^{\mathrm{a}}$ Helena Vila Suárez. Facultad de Ciencias de la Educación y del Deporte. Campus A Xunqueira s/n. C.P.: 36900. Universidad de Vigo, Pontevedra (España). E-mail: evigo@uvigo.es 
El envejecimiento es un proceso biológico, natural que ocurre en cualquier ser vivo, estos cambios tienen un carácter universal, donde se produce una disminución de las capacidades físicas (fuerza, agilidad, resistencia, equilibrio, coordinación...), capacidades cognitivas, y capacidades funcionales (Mancilla, Ramos, y Morales, 2016). Dentro de este proceso de envejecimiento donde el cuerpo comienza a mostrar efectos involutivos, uno de los principales efectos, es el que supone una pérdida de masa muscular que se relaciona con la pérdida de fuerza, es conocida como la sarcopenia (Sayer y Kirkwood, 2015) es un proceso que se acentúa con el de sedentarismo, pero aún en población físicamente activa este proceso avanza con la edad.

Un factor principal para que esto se produzca es el desuso del músculo esquelético, que acaba por producir una atrofia. La realización de actividad física regular constituye un hábito saludable, está demostrado que puede prevenir ciertas enfermedades, puede ayudar a aliviar los síntomas de la enfermedad en adultos mayores (García-Hermoso et al., 2018).

Además, se ha encontrado que la actividad física regular está asociada con una mejor calidad de vida entre los adultos mayores (Vagetti et al., 2014). El papel de la fuerza muscular en el desempeño de las actividades de la vida diaria y el ejercicio, así como en la prevención de enfermedades crónicas, se reconoce cada vez más (García-Hermoso et al., 2018).

Existen estudios que han correlacionado bajos niveles de masa muscular en personas mayores con una disminución de la calidad de vida (McLean y Kiel, 2015; Reid, Naumova, Carabello, Phillips, y Fielding, 2008), una disminución de la función física (Frontera et al., 2000) y un aumento de la mortalidad (Landi et al., 2012).

Se ha demostrado que la fuerza de agarre es un buen indicador de variables relacionadas con la salud, y se puede utilizar como un identificador de dependencia, mortalidad, caídas... (Bohannon, 2008; Lino et al., 2016; Sayer et al., 2006; Sayer y Kirkwood, 2015; Wang y Chen, 2010).

Europa es un continente donde la esperanza de vida es elevada, esto supone una prosperidad en muchos ámbitos de la sociedad, lo que lleva implícito, que cada vez este continente tiene más población envejecida, y ello hace la necesidad de replantearse muchos aspectos que influyen en la estructura social y económica de cada país.

A su vez, la población mayor cada vez es más activa en este proceso de envejecimiento, dispone de recursos y solicita su lugar dentro de la sociedad. Lo que hace necesario avanzar en estudios que aporten datos de las características de estas poblaciones, que ayuden a tomar decisiones y a elaborar estrategias para lograr una mayor calidad de vida. En la Unión Europea, ya existen precedentes en el que ante un reto o problema que afecta a varios de sus países el ser capaz de coordinarse para buscar soluciones en conjunto y ajustarlas a las características de esta sociedad. 
Dentro de este contexto, nace el proyecto Europeo "IN COMMON SPORTS" de Erasmus plus (IN COMMON SPORTS, 2018-2020), donde participan cinco países europeos (Hungría, Bulgaria, Italia, Portugal y España). Este proyecto consiste en acercar y familiarizar a las personas adultas mayores con la práctica de la actividad física y el deporte, por lo que pueden participar en el proyecto cualquier persona mayor que lo desee, y resida en las ciudades o cercanías donde se realizan las actividades ofertadas por el proyecto (ejercicio físico multidisciplinar). El objetivo de este estudio fue analizar los niveles de fuerza del tren superior con la calidad de vida de la población europea mayor de 65 años.

\section{MÉTODO}

\section{Participantes}

Han participado un total de 419 personas adultos mayores que residen en Bulgaria, Hungría, Portugal, Italia y España. Todos ellos pertenecientes al proyecto Europeo Erasmus plus, denominado "IN COMMON SPORTS". Un total de 419 adultos mayores, pertenecientes a cinco países europeos participaron en el estudio (Tabla 1). La media de edad estuvo comprendida entre los $68.02 \pm 11.46$ años de la población italiana hasta los $71.21 \pm 6.67$ años de la población española.

Los criterios de inclusión que se tuvieron en cuenta, fueron los mismos que se utilizaron para la realización del proyecto Europeo: que fuesen personas mayores, y que residieran en la ciudad que se implantaba el programa, o en las cercanías a dicha ciudad. Todos los participantes fueron informados de las pruebas que se iban a realizar y firmaron el consentimiento informado para participar en este proyecto. El estudio sigue los criterios de la declaración de Helsinki de 1964.

\section{Instrumentos}

Las medidas antropométricas fueron siguiendo los protocolos de la ISAK (International Working Group of Kinanthropometry) (Ross y Marfell-Jones, 1995). Para el peso se utilizó la Tanita TBF300 de $0.1 \mathrm{~kg}$ de precisión. Para la altura se utilizó el estadiometro Handac de 1,00 mm de precisión. Para el estudio de la composición corporal se calculó el porcentaje graso a través del índice de masa corporal (IMC), utilizando la siguiente fórmula: peso/talla2 $(\mathrm{kg} / \mathrm{m} 2)$. El porcentaje graso estimado por bioimpedancia (Tanita TBF300).

La fuerza de agarre del tren superior, se ha registrado a través de un dinamómetro hidráulico modelo SH5001 Saehan Corporation (Corea). Los participantes se encuentran en bipedestación, con el codo en flexión de $90^{\circ}$ y separado ligeramente del tronco (Mancilla, Ramos, y Morales, 2016). Se realizan 3 mediciones por cada extremidad y se calcula la media. Se utilizó el mejor de los tres intentos para cada mano. 
El cuestionario EuroQoL-5D es un instrumento que valora la calidad de vida relacionada con la salud (Herdman, Badia, y Berra, 2001). Se divide en 2 secciones. La primera sección contiene 5 preguntas sobre movilidad, cuidado personal, actividades cotidianas, dolor/malestar y ansiedad/depresión. Cada pregunta tiene tres niveles de gravedad (sin problemas, algunos problemas o problemas extremos). La segunda parte es una escala visual analógica de evaluación más general de su estado de salud, donde se registra la respuesta que puede valorar desde un 0 , donde es la peor salud imaginable y un 100 como la mejor salud imaginable.

\section{Procedimiento}

Para la realización del estudio se realizó una valoración a los 12 meses de comenzar el proyecto Europeo "IN COMMON SPORTS", donde se registraron las variables de antropometría y fuerza. Esta valoración se realizó de forma simultánea en todos los países participantes, y los instrumentos para la recogida de datos eran los mismos en todos los países. Además los participantes cubrieron una hoja con las actividades físicas que realizaban a la semana, tanto la frecuencia y la duración de estas, como la modalidad de actividad física que practicaban.

\section{Análisis estadístico}

Se ha llevado a cabo un análisis descriptivo a través de medidas de tendencia central (media y desviación típica/estándar) y porcentajes de las variables analizadas. Se ha comprobado la normalidad de las variables objeto de estudio a través de la prueba Kolmogorov-Smirnov ( $p>0.05)$. El análisis correlacional se llevó a cabo a través del coeficiente correlacional de Pearson. Los análisis estadísticos fueron llevados a cabo mediante el paquete estadístico IBM-SPSS v21 para Windows. La significatividad fue considerada para $p<0.05$.

\section{RESULTADOS}

Un total de 419 adultos mayores, pertenecientes a cinco países europeos participaron en el estudio (Tabla 1). El índice de masa corporal (IMC) estuvo comprendido entre los $27,80 \pm 6,24 \mathrm{~kg} / \mathrm{m} 2$ de la población italiana hasta los $30.32 \pm 4.54 \mathrm{~kg} / \mathrm{m} 2$ de la población búlgara. Para el porcentaje graso los mayores valores son los presentados por la población búlgara $38.26 \pm 6.67 \%$ frente a la población española $26.34 \pm 14.98 \%$. 
VILA et al. Fuerza muscular y calidad de vida en adultos mayores

Tabla 1. Características de la muestra del estudio por países

\begin{tabular}{|c|c|c|c|c|c|c|c|c|c|c|}
\hline & \multicolumn{10}{|c|}{ Países } \\
\hline & \multicolumn{2}{|c|}{ Bulgaria } & \multicolumn{2}{|c|}{ Hungría } & \multicolumn{2}{|c|}{ Italia } & \multicolumn{2}{|c|}{ Portugal } & \multicolumn{2}{|c|}{ España } \\
\hline & Media & $D T$ & Media & $D T$ & Media & $D T$ & Media & $D T$ & Media & $D T$ \\
\hline$\overline{\text { Edad (Años) }}$ & 70.14 & 7.78 & 66.42 & 5.94 & 68.02 & 11.46 & 69.64 & 9.93 & 71.21 & 6.67 \\
\hline EQ5D5L, Movilidad & 2.08 & .95 & 1.44 & .76 & 1.48 & .88 & 1.36 & .59 & 1.62 & .84 \\
\hline EQ5D5L, Cuidado personal & 1.71 & .96 & 1.05 & .40 & 1.10 & .43 & 1.05 & .21 & 1.12 & .52 \\
\hline EQ5D5L, Actividades Cotidianas & 2.07 & .97 & 1.13 & .47 & 1.25 & .61 & 1.11 & .38 & 1.26 & .54 \\
\hline EQ5D5L, Dolor & 2.43 & .88 & 1.47 & .74 & 1.86 & .79 & 1.71 & .94 & 1.98 & .84 \\
\hline EQ5D5L, Ansiedad & 2.18 & 1.05 & 1.22 & .53 & 1.74 & .92 & 1.47 & .84 & 1.55 & .85 \\
\hline EQ5D5L, Estado salud & 65.12 & 16.03 & 81.78 & 12.05 & 77.07 & 15.51 & 78.39 & 17.66 & 73.15 & 20.63 \\
\hline IMC $\left(\mathrm{kg} / \mathrm{m}^{2}\right)$ & 30.32 & 4.54 & 29.27 & 7.50 & 27.80 & 6.24 & 28.19 & 4.30 & 30.26 & 5.92 \\
\hline$\%$ Graso & 38.26 & 6.67 & 37.04 & 7.42 & 29.05 & 8.34 & 35.59 & 9.12 & 26.34 & 14.98 \\
\hline Fuerza de agarre Izq. (kg.) & -- & -- & 26.69 & 8.36 & 27.65 & 10.53 & 27.09 & 10.41 & 28.24 & 7.86 \\
\hline Fuerza de agarre Drch (kg.) & -- & -- & 27.34 & 8.16 & 30.13 & 10.51 & 28.31 & 10.33 & 29.72 & 6.95 \\
\hline
\end{tabular}

De los 419 participantes, 317 fueron mujeres y 102 hombres (Tabla 2). El mayor porcentaje de la muestra corresponde a la población italiana. La edad media de las mujeres fue de $69.12 \pm 49.04$ años frente a los $70.23 \pm 9.57$ años de los hombres. Las mujeres presentan un estado de salud, un IMC y la fuerza de agarre (derecha e izquierda) menor que los hombres.

Tabla 2. Características de la muestra por género y frecuencia de los participantes por países

\begin{tabular}{|c|c|c|c|c|c|}
\hline & & \multicolumn{2}{|c|}{ Mujeres $n=317$} & \multicolumn{2}{|c|}{ Hombres $n=102$} \\
\hline & & Media / \% & $D T$ & Media / \% & $D T$ \\
\hline Edad (Años) & & 68.76 & 9.04 & 70.23 & 9.57 \\
\hline \multirow{5}{*}{ País } & Bulgaria & $20.2 \%$ & & $11.8 \%$ & \\
\hline & Hungría & $13.6 \%$ & & $11.8 \%$ & \\
\hline & Italia & $30.3 \%$ & & $26.5 \%$ & \\
\hline & Portugal & $16.4 \%$ & & $32.4 \%$ & \\
\hline & España & $19.6 \%$ & & $17.6 \%$ & \\
\hline EQ5D5L, Movilidad & & 1.64 & 0.89 & 1.39 & 0.72 \\
\hline EQ5D5L, Cuidado Personal & & 1.23 & 0.65 & 1.11 & 0.41 \\
\hline EQ5D5L, Actividades cotidianas & & 1.40 & 0.76 & 1.23 & 0.55 \\
\hline EQ5D5L, Dolor & & 1.98 & 0.92 & 1.65 & 0.74 \\
\hline EQ5D5L, Ansiedad & & 1.72 & 0.94 & 1.49 & 0.84 \\
\hline EQ5D5L, Estado de salud & & 74.13 & 17.59 & 78.18 & 16.36 \\
\hline IMC $\left(\mathrm{kg} / \mathrm{m}^{2}\right)$ & & 29.31 & 6.30 & 27.80 & 3.42 \\
\hline$\%$ Graso & & 35.26 & 9.74 & 24.18 & 9.66 \\
\hline Fuerza agarre Izq. (kg.) & & 24.17 & 8.63 & 37.95 & 9.27 \\
\hline Fuerza agarre Drch. (kg.) & & 25.60 & 6.45 & 38.93 & 8.95 \\
\hline
\end{tabular}

Nota. $D T=$ desviación típica. Izq= izquierda; Drch $=$ derecha

El análisis correlacional efectuado ha desvelado que cinco áreas de la calidad de vida (movilidad, cuidado personal, actividades cotidianas, dolor/malestar y ansiedad/depresión) han presentado una correlación muy significativa e inversa $(p<0.001)$ con la fuerza isométrica del tren superior. Respecto al estado de salud general se han presentado correlaciones muy significativas y directas con la fuerza del tren superior (ver Tabla 3). 
Tabla 3. Análisis correlacional entre la Fuerza de agarre derecha e izquierda con la Calidad de Vida

\begin{tabular}{llcc}
\hline & & $\begin{array}{c}\text { F. Agarre Drch. } \\
(\mathrm{kg} .)\end{array}=386$ & $\begin{array}{c}\text { F. Agarre Izq. (kg.) } \\
n=373\end{array}$ \\
\hline \multirow{2}{*}{ EQ5D5L, Movilidad } & Correlación de Pearson & $-.297^{* *}$ & $-.308^{* *}$ \\
\cline { 2 - 4 } & Sig. (bilateral) & .001 & .001 \\
\hline \multirow{2}{*}{ EQ5D5L, Cuidado Personal } & Correlación de Pearson & $-.306^{* *}$ & $-.319^{* *}$ \\
\cline { 2 - 4 } & Sig. (bilateral) & .001 & .001 \\
\hline \multirow{2}{*}{ EQ5D5L, Actividades cotidianas } & Correlación de Pearson & $-.374^{* *}$ & $-.387^{* *}$ \\
\cline { 2 - 4 } & Sig. (bilateral) & .001 & .001 \\
\hline \multirow{2}{*}{ EQ5D5L, Dolor } & Correlación de Pearson & $-.292^{* *}$ & $-.298^{* *}$ \\
\cline { 2 - 4 } & Sig. (bilateral) & .001 & .001 \\
\hline \multirow{2}{*}{ EQ5D5L, Ansiedad } & Correlación de Pearson & $-.264^{* *}$ & $-.264^{* *}$ \\
\cline { 2 - 4 } & Sig. (bilateral) & .001 & .001 \\
\hline \multirow{2}{*}{ EQ5D5L, Estado de salud } & Correlación de Pearson & $.303^{* *}$ & $.305^{* *}$ \\
\cline { 2 - 4 } & Sig. (bilateral) & .001 & .001 \\
\hline
\end{tabular}

Nota. $\mathrm{F}=$ fuerza; Izq= izquierda; Drch= derecha

Respecto a la calidad de vida y la fuerza de agarre, el mayor valor de la correlación indica que a mayores niveles de fuerza de agarre mejores valoraciones del estado de salud (Gráfica 1).

Gráfica 1. Correlación entre la fuerza de agarre con el estado de salud

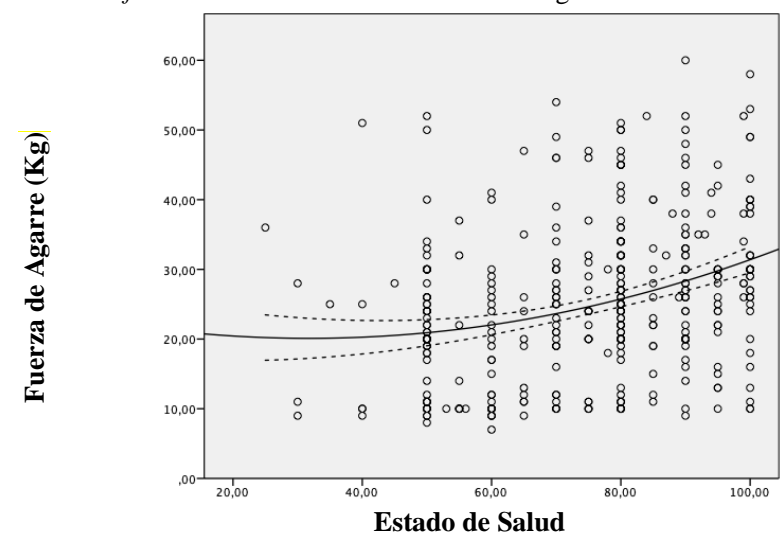

\section{DISCUSIÓN Y CONCLUSIONES}

Los resultados constatan una relación inversa entre calidad de vida y fuerza de tren superior, es decir, mayores valores de fuerza de tren superior se relacionan con mejor calidad de vida para la población europea mayor de 65 años. Estos resultados están en línea con los presentados por otros estudios (Bohannon, 2008; Lino et al., 2016; Reid et al., 2008; Sayer et al., 2006; Wang y Chen, 2010).

Las poblaciones de mayor edad son la española y la búlgara, sobrepasan los 70 años, y a su vez presentan obesidad, mientras que la población de los otros tres países 
está situadas en el sobrepeso. Son los búlgaros los que presentan puntuaciones más elevadas para todas las variables del cuestionario de calidad de vida, incluido el estado de salud, que lo valoran con una media de 65.12 puntos sobre 100. En el otro extremo se encuentran los húngaros e italianos con las menores puntuaciones en el cuestionario de calidad de vida (por lo tanto más autónomos son) y las mejores valoraciones medias en su estado de salud, 81.78 los búlgaros y 78.39 los italianos.

Por género, las mujeres europeas son más jóvenes que los hombres, pero presentan valores más elevados en las valoraciones del cuestionario de calidad de vida y estado de salud con una puntuación inferior a la de los hombres (74.13 vs 78.18 respectivamente). Respecto a los valores presentados en otros estudios con adultos sanos, los valores de la fuerza de agarre de la población de este estudio han sido superiores a las presentadas por Mancilla et al. (2016) y Lino et al. (2016), con población chilena y brasileña respectivamente y en ambos géneros.

Siguiendo a Cruz-Jentoft et al., (2010), la población analizada también presenta más de $10 \mathrm{~kg}$ de diferencia entre géneros (en este estudio es menor a $10 \mathrm{Kg}$ ), y los valores medios que presenta en la fuerza de agarre en ambas manos son superiores a los propuestos por ellos para ser diagnosticados con sarcopenia. Respecto a la dominancia, en este estudio la población presenta más fuerza en la mano derecha que en la izquierda, las diferencias no son grandes, esto coincide con otras poblaciones (Mancilla et al., 2016; Lino et al., 2016).

Las cinco partes de las que se compone el cuestionario sobre la calidad de vida, presentan correlaciones muy significativas con la fuerza de agarre de ambas manos, así como también con una escala de como el sujeto percibe su estado de salud. Los resultados de este estudio mantienen la línea de identificar la fuerza de agarre como un indicador fiable para prevenir limitaciones funcionales futuras, y un mejor estado físico (Cruz-Jentoft et al., 2010).

Por ello se puede identificar la fuerza de agarre como un indicador que se relaciona con la calidad de vida, estando en línea con otros estudios (Sayer et al., 2006; Gum et al., 2018; Wang y Chen, 2010) aunque utilizan diferentes instrumentos para analizar la calidad de vida.

Basándonos en los resultados de este estudio, teniendo presente que es un estudio descriptivo, como principal conclusión se puede confirmar que la fuerza de agarre correlaciona de manera positiva con la calidad de vida. Esta es una variable de fácil y rápida medición, sería recomendable plantearse el incorporarla en los diferentes protocolos de valoración que se planten realizar a la población adulta mayor. Son numeroso los estudios que presentan evidencias de su validez. 


\section{REFERENCIAS}

Bohannon, R.W. (2008). Hand-grip dynamometry predicts future outcomesin aging adults. Journal of Geriatric Physical Therapy, 31, 3-10.

Cruz-Jentoft, A.J., Baeyens, J.P., Bauer, J.M., Boirie, Y., Cederholm, T., Landi, F., ... Vandewoude, M. (2010). Sarcopenia: European consensus on definition and diagnosis: Report of the European Working Group on Sarcopenia, in Older People. Age Ageing, 39, 412-423.

Frontera, W.R., Suh, D., Krivickas, L.S., Hughes, V.A., Goldstein, R., y Roubenoff, R. (2000). Skeletal muscle fiber quality in older men and women. American Journal of Physiology Cell Physiology, 279, C611-618.

García-Hermoso, A., Cavero-Redondo, I., Ramírez-Vélez, R., Ruiz, J.R., Ortega, F.B., Lee, D.C., y Martínez-Vizcaíno, V. (2018). Muscular strength as a predictor of all-cause mortality in an apparently healthy population: a systematic review and meta-analysis of data from approximately 2 million men and women. Archives of Physical Medicine and Rehabilitation, 99, 2100.e5-2113.e5.

Gum, A.M., Segal-Karpas, D., Avidor, S., Ayalon, L., Bodner, E., y Palgi, Y. (2018). Grip strength and quality of life in the second half of life: hope as a moderator. Aging Ment Health, 22(12), 1600-1605.

Herdman, M., Badia, X., y Berra, S. (2001). El EuroQol-5D: una alternativa sencilla para la medición de la calidad de vida relacionada con la salud en atención primaria. Atención Primaria, 28(6), 425-429.

Landi, F., Liperoti, R., Fusco, D., Mastropaolo, S., Quattrociocchi, D., Proia, A., ... Onder, G. (2012). Sarcopenia and mortality among older nursing home residents. Journal of the American Medical Directors Association, 13, 121-126.

Lino, V.T.S., Rodrigues, N.C.P., O’Dwyer, G., Andrade, M.K.dN., Mattos, I.E., y Portela, M.C. (2016). Handgrip strength and factors associated in poor elderly assisted at a primary care unit in Rio de Janeiro, Brazil. PLoS ONE, 11(11), e0166373.

Mancilla, E., Ramos, S., y Morales, P. (2016). Fuerza de prensión manual según edad, género y condición funcional en adultos mayores Chilenos entre 60 y 91 años. Revista Médica de Chile, 144(5), 598-603.

McLean, R.R., y Kiel, D.P. (2015). Developing consensus criteria for sarcopenia: An update. Journal of Bone and Mineral Research, 30(4), 588-592.

Reid, K.F., Naumova, E.N., Carabello, R.J., Phillips, E.M., y Fielding, R.A. (2008). Lower extremity muscle mass predicts functional performance in mobilitylimited elders. Journal Nutricion Health Aging, 12, 493-498.

Ross, W.D., y Marfell-Jones, R.J. (1995). Cinantropometria. In J. Duncan, H. MacDougall, A. Wenger, y H.J. Green (Eds.), Evaluación fisiológica del deportista. Barcelona: Paidotribo.

Sayer, A.A., Syddall, H.E., Martin, H.J., Dennison, E.M., Roberts, H.C., y Cooper, C. (2006). Is grip strength associated with health-related quality of life? Findings from the Hertfordshire Cohort Study. Age and Ageing, 35(4), 409-415.

Sayer, A.A., y Kirkwood, T.B. (2015). Grip strength and mortality: a biomarker of ageing? Lancet, 386, 226-227.

Vagetti, G.C., Barbosa, V.C., Moreira, N.B., Oliveira, V., Mazzardo, O., y Campos, W. (2014). Association between physical activity and quality of life in the elderly: a systematic review, 2000-2012. Revista Brasileira de Psiquiatría, 36(1), 76-88. 
Wang, C.Y., y Chen, L.Y. (2010). Grip strength in older adults: test-retest reliability and cutoff for subjective weakness of using the hands in heavy tasks. Archives of Physical Medicine and Rehabilitation, 91(11), 1747-1751.

Recibido: 20 de septiembre de 2019

Recepción Modificaciones: 29 de noviembre de 2019

Aceptado: 2 de diciembre de 2019 